\title{
Ultra-flat bismuth films for diamagnetic levitation by template-stripping
}

\author{
J. Kokorian a,b, J.B.C. Engelen a,c J. de Vries ${ }^{\text {a }}$, H. Nazeer ${ }^{\text {a }}$, L.A. Woldering ${ }^{\text {a }}$, L. Abelmann ${ }^{\text {a,* }}$ \\ a University of Twente, P.O. Box 217, 7500 AE Enschede, The Netherlands \\ b TU Delft - 3mE-PME, Mekelweg 2, 2628 CD Delft, The Netherlands \\ c IBM Research - Zurich, Säumerstrasse 4, CH-8803 Rüschlikon, Switzerland
}

\section{A R T I C L E I N F O}

\section{Article history:}

Received 20 February 2013

Received in revised form 10 November 2013

Accepted 14 November 2013

Available online 23 November 2013

\section{Keywords:}

Bismuth

Template stripping

Surface roughness

Crystal structure

Young's modulus

\begin{abstract}
A B S T R A C T
In this paper we present a method to deposit thin films of bismuth with sub-nanometer surface roughness for application to diamagnetic levitation. Evaporated films of bismuth have a high surface roughness with peak to peak values in excess of $100 \mathrm{~nm}$ and average values on the order of $20 \mathrm{~nm}$. We expose the smooth backside of the films using a template stripping method, resulting in a great reduction of the average surface roughness, to $0.8 \mathrm{~nm}$. Atomic force microscope and X-ray diffraction measurements show that the films have a polycrystalline texture with preferential c-axis orientation. On the back side of the film, fine grains are grouped into larger clusters. Cantilever resonance shift measurements indicate that the Young's modulus of the films is on the order of $20 \mathrm{GPa}$.
\end{abstract}

(c) 2013 Published by Elsevier B.V.

\section{Introduction}

Diamagnets possess the fascinating property that they can be stably positioned in non-uniform magnetic fields, without dissipation of energy [1]. Superconductors for instance are diamagnets with magnetic susceptibility of -1 , and find important applications in, for example, levitated trains [2]. Here, even though no energy is required for stable levitation, energy is dissipated to maintain the superconducting temperature. Room temperature diamagnets have much smaller diamagnetic constants. Silicon has a susceptibility of $-3.4 \cdot 10^{-6}$, water has a susceptibility of $-10^{-6}$, and even the highest known room-temperature diamagnets have susceptibilities only on the order of $-10^{-4}$ [3]. Thus, in room temperature levitation, the levitating forces are orders of magnitude lower than in a superconducting train. As a consequence, room temperature levitation at dimensions in the meter range is not possible.

Room temperature levitation is possible however if we shrink the dimensions. The diamagnetic force density $f_{\mathrm{d}}$ is proportional to the gradient of the magnetic field $\mathbf{H}$ [4],

$f_{\mathrm{d}}=\mu_{0} \chi|\boldsymbol{H}| \nabla(|\boldsymbol{H}|)$

where $\mu_{0}$ is the permeability of free space and $\chi$ is the volume magnetic susceptibility of the material.

The gradient is inversely proportional to the dimensions of the system. Consequently, the smaller the system, the larger the gradient and the larger the diamagnetic force densities. The development of

\footnotetext{
* Corresponding author.

E-mail address: l.abelmann@utwente.nl (L. Abelmann).
}

high-field permanent magnets has enabled dissipation-free levitation of millimeter-sized objects. Today, one can buy toys where a thin highly-oriented pyrolytic graphite (HOPG) disk of about $1 \mathrm{~mm}$ thickness and $10 \mathrm{~mm}$ diameter is levitated above an array of four strong rare-earth NdFeB magnets with a magnetization of about $1.4 \mathrm{~T}-1.6 \mathrm{~T}$ (Grand Illusions). Even milliliters of water (the main constituent of tiny frogs) can be levitated against the gravitational field in the $40 \mathrm{~mm}$ bore of a $16 \mathrm{~T}$ magnet [5].

When moving to micrometer sized magnets, the field gradients increase again by orders of magnitude. In this regime, it is in principle possible to levitate magnets above diamagnetic substrates. This opens up a range of wireless, frictionless actuation principles. Diamagnetic levitation is used for low-friction microelectromechanical systems, such as accelerometers [6], gyroscopes and inclinometers [7]. To enable these applications, materials with high diamagnetic constants as well as micrometer-size rare-earth permanent magnets were required $[4,8]$.

The research presented in this paper is aimed at the development of a levitation system where a micro-magnet is levitated above an ultra-smooth diamagnetic substrate at a height of around 1 , as is illustrated in Fig. 1. A small magnet that levitates above a diamagnetic substrate will always move towards a minimum of the magnetic field. In the absence of an external magnetic field, the micro-magnet is levitated entirely because of its own field and the interaction of its field with the diamagnetic substrate. Irregularities in the diamagnetic substrate will influence the magnetic field and as a result the magnet will wiggle from one magnetic minimum to another magnetic minimum, greatly deteriorating the predictability of its dynamic behavior. When the substrate is perfectly flat, the levitating micro-magnet will have no preferential position, because over the entire area of the diamagnetic substrate, the magnet is located on an energetic saddle 


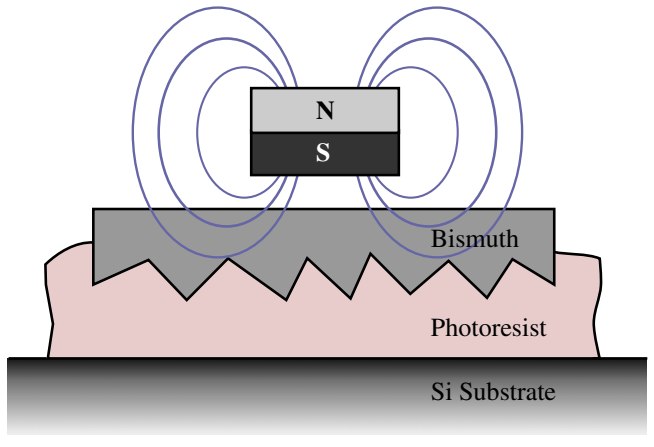

Fig. 1. Impression of a micro-magnet levitating above the flat backside of a templatestripped bismuth film (not to scale). The large roughness of the former topside of the bismuth film introduces magnetic minima. Therefore, a template-stripping method was used to put the smooth backside of the film on top.

point. It is therefore desirable to use a diamagnetic substrate that is as smooth as possible.

Unfortunately, materials with both a high diamagnetic constant and a low surface roughness are not easily obtainable. The material with the highest known diamagnetic constant at room temperature is highlyoriented pyrolytic graphite (HOPG). However, the high-pressure chemical vapor deposition fabrication method of HOPG [9] does not allow fabrication of smooth films that are several hundreds of nanometers thick, which is required for diamagnetic levitation.

Therefore, we have directed our attention to bismuth, which has the largest diamagnetic constant of all metals, $\chi=-165 \cdot 10^{-6}[3]$. The melting temperature of bismuth is very low $\left(271^{\circ} \mathrm{C}\right)$. As a result, bismuth grows as a very rough polycrystalline film, regardless of whether the film is deposited by pulsed laser deposition [10], sputtering [11,12], or thermal evaporation [13-15]. Deposited bismuth films have a high roughness, which increases with the film thickness. For substrate temperatures higher than about 200, the films are discontinuous and consist of large droplets. Epitaxially grown ultra-thin films of bismuth with a root mean square (RMS) surface roughness of $0.6 \mathrm{~nm}$ have been produced [15], but only up to a film thickness of $25 \mathrm{~nm}$. This thickness is too thin for practical application in diamagnetic levitation. A study by T. Missana et al. [12] has shown that the surface texture of films up to $100 \mathrm{~nm}$ thick can be improved by pulsed laser melting, but no quantitative measurements are shown regarding the surface roughness.

Rather than trying to obtain smooth deposited surfaces, we employed a template stripping method that is commonly used for the fabrication of ultra-smooth thin films of gold [16]. By gluing an additional bonding substrate on top of the deposited film and tearing the two wafers apart again, the film remains glued to the bonding wafer and is released from the substrate it was deposited on. The result is illustrated in Fig. 1, which shows a levitating magnet above the smooth diamagnetic bismuth layer film.

In the following text, we investigate the surface roughness of template-stripped bismuth films using high-resolution scanning electron microscopy (SEM) and atomic force microscopy (AFM). In order to determine the causes for surface roughness, both on the front and backside of the film, we investigate their crystal texture by X-ray diffractometry (XRD). Furthermore, we determine mechanical properties such as Young's modulus and residual stress of the thin to gain deeper inside in the film's texture and structure.

\section{Experimental details}

The template stripping process is outlined in Fig. 2. First, a $1.7 \mu \mathrm{m}$ thick layer of OLIN907/17 photoresist is spin-coated on top of the bismuth film after priming it with a hexamethyldisilazane (HMDS) primer. The bismuth substrate wafer is then bonded with an HMDS primed silicon wafer by the 'glue' layer of photoresist and is left to cure overnight at $50{ }^{\circ} \mathrm{C}$ with a $500 \mathrm{~g}$ weight on top. The bismuth can then be stripped from the substrate wafer by carefully forcing a pair of flat tweezers in between the bonded wafers.

The bismuth is deposited in a Balzers BAK-600 batch evaporation system with a Maxtek MDC-360C in-situ deposition rate and thickness monitor. We used 99.999\% pure bismuth pieces (Kurt J. Lesker) as evaporation material. The substrates we used were plain silicon (100) wafers. Before loading the wafers into the vacuum chamber, the native silicon oxide was removed by a brief $1 \% \mathrm{HF}$ wet-etch. We then deposited $200 \mathrm{~nm}$ and $500 \mathrm{~nm}$ thick bismuth films at rates around $8 \mathrm{~nm} \mathrm{~s}^{-1}$ and $5 \mathrm{~nm} \mathrm{~s}^{-1}$, respectively. The background pressure during deposition was $3 \times 10^{-5} \mathrm{~Pa}$. The temperature of the substrates could not be measured during deposition. Given the low crucible temperature, short deposition time, large mass of the substrate holder, and the fact that the deposition chamber was cooled, it is very unlikely that the substrate temperatures rose above $60{ }^{\circ} \mathrm{C}$.

We carried out XRD measurements on both films in a Philips XRD Expert system II with a Cu-K alpha X-ray source. A $2 \theta-\omega$ scan was performed from $20^{\circ}$ to $90^{\circ}$ in steps of 0.01 every $2.5 \mathrm{~s}$. The X-ray source acceleration voltage was set at $40 \mathrm{kV}$ with a current of $30 \mathrm{~mA}$. We measured a rocking curve around the (003) peak of the XRD patterns with a range of $30^{\circ}$ in steps of $0.005^{\circ}$ every $2.5 \mathrm{~s}$. The peaks in the XRD pattern where identified using the PDF 00-005-0519 database.

Both the topside and backside of the deposited bismuth films were analyzed by an FEI Quanta 3D Dualbeam FEGSEM/FIB and a DI3100 AFM in tapping mode with proportional-integral feedback.

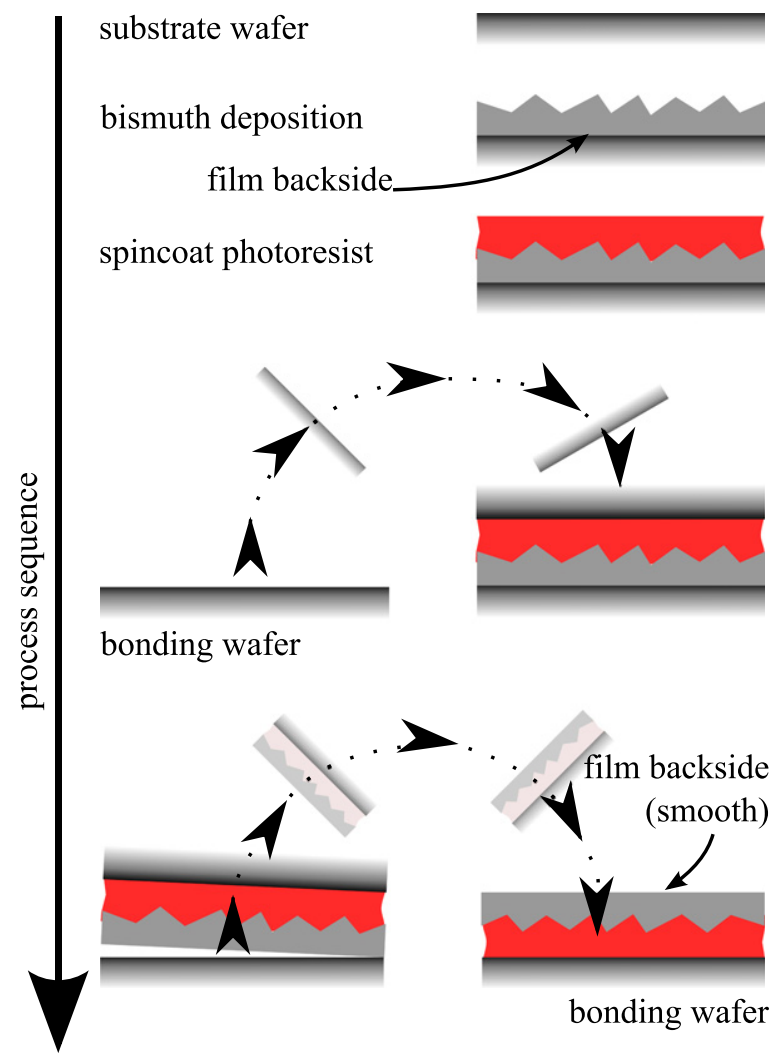

Fig. 2. Schematic diagram of the template stripping process. First bismuth is deposited on a silicon substrate. A layer of photoresist is spincoated on top of the bismuth film and a bonding wafer is stuck to it. Using mechanical force, the wafers are then separated in the course of which the bismuth is released from the substrate wafer and sticks to the photoresist. The smooth former backside of the film is now exposed. 


\section{Determination of Young's modulus and residual stress}

In addition to surface characterization with AFM and SEM and crystallographic analysis by XRD, we use the mechanical properties to gain deeper inside in the texture and structure of the thin Bismuth film.

The Young's modulus of thin films can be determined from the change in resonance frequencies of a set of cantilevers before and after the deposition of a film on top of the cantilevers [17]. For this purpose a $200 \mathrm{~nm}$ thick bismuth film was deposited on an array of silicon cantilevers in the same run as the Si wafers. These cantilevers were fabricated from silicon-on-insulator wafers with a $3 \mu \mathrm{m}$ thick device layer, using a deep reactive ion etching process [18]. The resonance frequencies of the cantilevers were measured with a Polytec MSA-400 scanning laser-Doppler vibrometer before and after deposition of the bismuth film.

The thin film on the cantilever affects its flexural rigidity and increases its mass, which results in a change of its resonance frequency. The relation between the Young's modulus and resonance frequency shift is taken from Nazeer et al. [18]. This theory is based on a shift of the cantilever's neutral axis and on the assumptions that the material is linearly elastic, that the cantilever has a uniform cross-section, and that its deflection is small compared to the length. For the calculation, the Young's modulus of silicon is taken as $168.9 \mathrm{GPa}$ [19].

The cantilevers are also bent due to the residual stress in the deposited film. From the cantilever deflection, the residual stress can be readily determined using Stoney's equation [20],

$\sigma_{\mathrm{f}}=\frac{1}{3} \frac{E_{\mathrm{c}} t_{\mathrm{c}}^{2} \xi}{t_{\mathrm{f}} L^{2}}$

with residual stress $\sigma_{\mathrm{f}}$, cantilever Young's modulus $E_{\mathrm{c}}$, cantilever length $L$ and thickness $t_{\mathrm{c}}$, film thickness $t_{\mathrm{f}}$ and cantilever deflection $\xi$.

Because an accurate value of the bismuth film thickness is required for the determination of the Young's modulus, we verified the film thickness by SEM imaging of the film fracture cross-sections, see Section 4.3.

\section{Results and discussion}

\subsection{Film surface texture}

We will refer to the two deposited bismuth films by their 'intended film thickness', i.e., the film thickness we intended them to have: $t_{d}=200 \mathrm{~nm}$ and $500 \mathrm{~nm}$. AFM measurements of the deposited films are shown in Fig. 3. Both the $200 \mathrm{~nm}$ and the $500 \mathrm{~nm}$ film have a rough surface with similar shaped grains. The surface roughness parameters determined from the AFM measurements are given in Table 1. Fig. 4 shows the histograms of the measured topography.

The structure of the films corresponds to the rough but continuous film structures reported by L. Kumari et al. [13] for films that were deposited at $30{ }^{\circ} \mathrm{C}$ and $100{ }^{\circ} \mathrm{C}$. Because we do not observe island formation, we conclude that the substrate temperature remained well below $200{ }^{\circ} \mathrm{C}$ during deposition.

\subsection{X-Ray diffraction measurements}

The XRD measurement results are shown in Fig. 5. The peaks in our XRD measurement results appear at the same $2 \theta$ angles as those measured by L. Kumari et al. [13]. Even though bismuth has a rhombohedral unit cell, especially in polycrystalline films, it is useful to convert it to a hexagonal unit cell [21]. In this case, one can attribute the observed peaks to the (003), (006) and (009) planes. The rocking curve measurements around the principal (003) peak at $2 \theta=22.5^{\circ}$ confirm that the films are polycrystalline with a preferential c-axis orientation. The small peak at $2 \theta=69.0^{\circ}$ originates from the (400) plane of the silicon substrate. The origin of the peak at $2 \theta=53.4^{\circ}$


Fig. 3. AFM images of the bismuth films deposited by thermal evaporation. Scan range: $5 \mu \mathrm{m} \times 5 \mu \mathrm{m}$. The images show that both films are of polycrystalline nature and have a large surface roughness. The maximum height difference is $140.5 \mathrm{~nm}$ for the $200 \mathrm{~nm}$ film and $241.2 \mathrm{~nm}$ for the $500 \mathrm{~nm}$ film.

remains unclear. It does not match any known scattering pattern of bismuth, bismuth oxide or silicon.

By applying Scherrer's equation, we determined that the average crystal size is $72 \mathrm{~nm}$ and $88 \mathrm{~nm}$ for, respectively, the $200 \mathrm{~nm}$ and $500 \mathrm{~nm}$ thick films. This is in agreement with the AFM observation of Fig. 3, where the average crystallite size appears to be on the same order of magnitude.

\subsection{Measurement of the film thickness}

Because the films are rough compared to their thickness, the definition of the film thickness is not trivial. We can identify the peaks from the cross-section SEM image, but it is much harder to identify small holes. We therefore suggest an 'apparent minimum thickness'. The

Table 1

Topography of the bismuth films' top sides determined from the AFM measurements shown in Fig. 3, where $t_{d}$ is the intended film thickness, $R_{a}$ is the arithmetic average of absolute values, $R_{p p}$ is the peak-to-peak roughness, i.e., the vertical distance from the lowest to the highest value of the height profile, and $\mathrm{R}_{\mathrm{RMS}}$ is the RMS roughness. The median and average denote the median and average of the AFM height profile.

\begin{tabular}{lcc}
\hline & $\mathrm{t}_{\mathrm{d}}=200 \mathrm{~nm}$ & $\mathrm{t}_{\mathrm{d}}=500 \mathrm{~nm}$ \\
\hline $\mathrm{R}_{\mathrm{a}}$ & $13.4 \mathrm{~nm}$ & $20.3 \mathrm{~nm}$ \\
$\mathrm{R}_{\mathrm{pp}}$ & $140.5 \mathrm{~nm}$ & $241.2 \mathrm{~nm}$ \\
$\mathrm{R}_{\mathrm{RMS}}$ & $17.4 \mathrm{~nm}$ & $25.5 \mathrm{~nm}$ \\
Median & $28.1 \mathrm{~nm}$ & $104.1 \mathrm{~nm}$ \\
Average & $34.8 \mathrm{~nm}$ & $109.7 \mathrm{~nm}$ \\
\hline
\end{tabular}


a

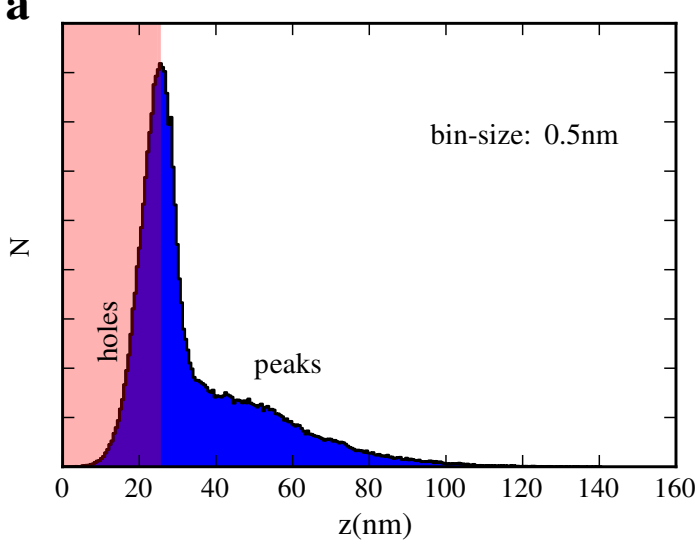

b

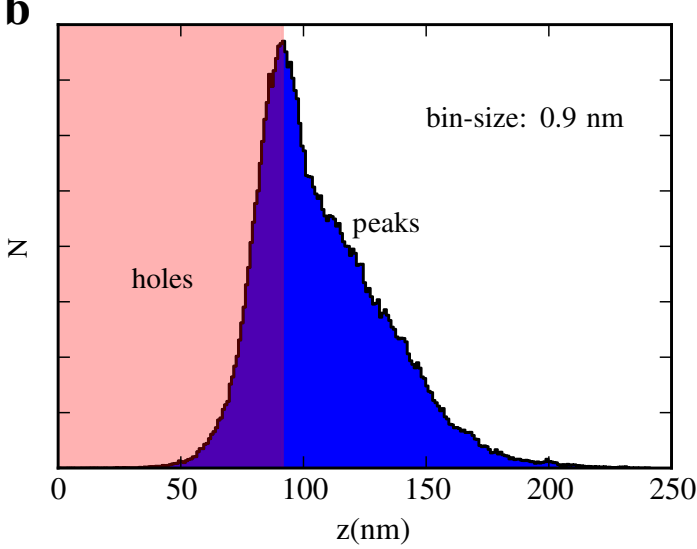

Fig. 4. Histograms of the height values $z$ of the AFM measurements shown in Fig. 3.

histogram of the $z$-values measured with the AFM has a narrow peak for lower values of $z$ and another bump for higher values of $z$. Comparing this histogram with the cross-section SEM reveals that the bump at higher $z$ values represents the peaks that protrude above the surface, which means that the left tail of the narrow peak at lower $z$-values represents the small amount of holes in the surface. The peak of the histogram itself then corresponds to the apparent minimum thickness. Fig. 6a and b show the cross-section SEM images of the $200 \mathrm{~nm}$ and $500 \mathrm{~nm}$ thick films, with an overlay of the histogram of the $z$-values measured with the AFM. The histogram is offset in such a way that the peak matches the apparent minimum thickness determined from the SEM images.

The average value of the offset AFM histogram now represents an 'effective' thickness that corresponds to the film thickness at which the amount of material in the peaks above the effective thickness is compensated by the amount of material 'missing' from the 'holes' beneath the effective thickness. Hence, the effective thickness represents the thickness of a hypothetical, perfectly flat film that contains the same amount of mass as the actual film. The film thickness values are summarized in Table 2 .

\subsection{Young's modulus and residual stress measurements}

The effective thickness of (176 \pm 13$) \mathrm{nm}$ is used for the calculation of the Young's modulus. By averaging the measured values of the Young's modulus on the different cantilevers, its mean value could be determined to be $(20 \pm 11)$ GPa. The large error is mainly caused by the uncertainty in the film thickness. The relation between the observed value for the Young's modulus and values reported for bulk crystal is complex [22], since it involves transformation of the rhombohedral unit cell into the hexagonal cell, and subsequent averaging over the
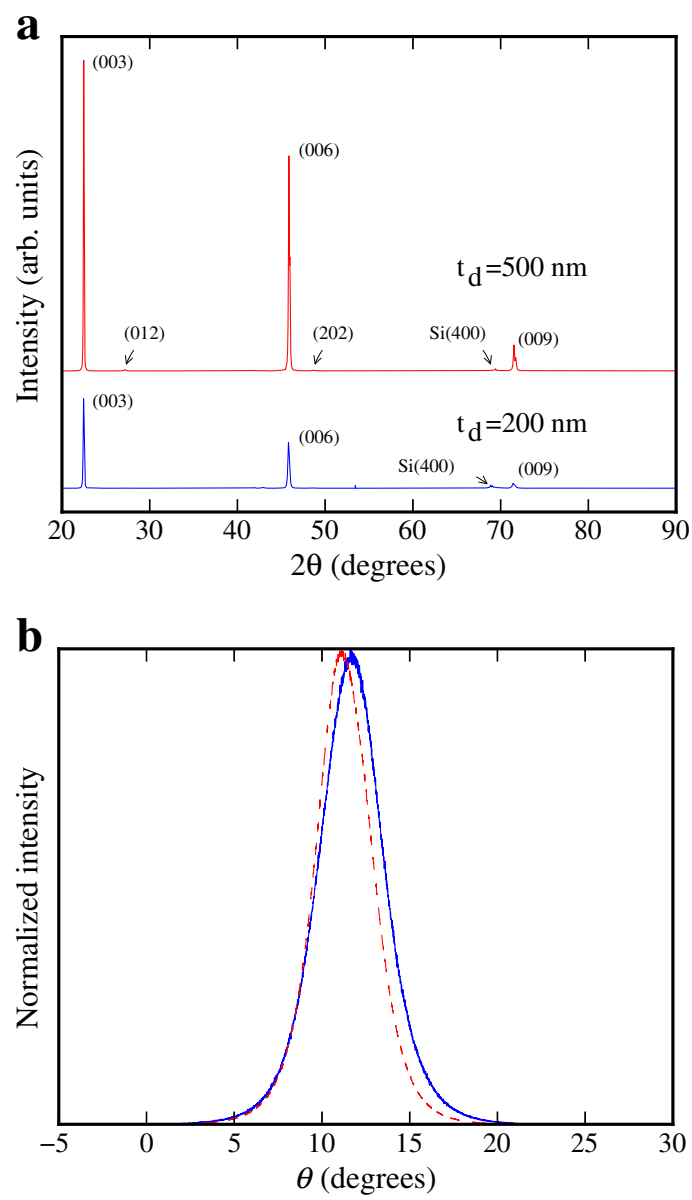

Fig. 5. XRD patterns and rocking curves of the $200 \mathrm{~nm}$ (dashed red) and $500 \mathrm{~nm}$ (solid blue) thick bismuth films.

in-plane orientation to correct for the polycrystalline nature of the film. The experimental value found here is however in the order of the values found for bulk bismuth crystals, which ranges from $63 \mathrm{GPa}$ for $E_{11}$ down to $7 \mathrm{GPa}$ for $E_{14}$ [23]. This result suggests that the grains in the film are connected in a similar way as in bulk material.

Fig. $7 \mathrm{~b}$ shows the residual stress obtained from the cantilever bending. Surprisingly, for longer cantilevers, we observe a reversal in the residual stress. Although shorter cantilevers bend upwards (tensile stress), longer cantilevers bend downwards (compressive stress). We have no convincing explanation for this effect, although we suspect that a difference in temperature between the cantilever and the substrate holder during growth might play a role.

\subsection{Template stripping}

The AFM measurements and SEM micrographs of the template stripped bismuth films are shown in Figs. 8 and 9 . The $500 \mathrm{~nm}$ thick film shows a structure of large, flat grains that is punctured with holes which are several nanometers deep. The $200 \mathrm{~nm}$ thick film appears to have a finer grain structure, but is punctured with small holes as well. Table 3 shows the statistical data from the AFM measurement. Both films have sub-nanometer RMS roughness.

\subsection{Bismuth film structure}

The AFM measurement of the $500 \mathrm{~nm}$ thick film reveals a structure of large grains. A zoom to a region of $1 \mu \mathrm{m} \times 1 \mu \mathrm{m}$, shown in Fig. 9c, reveals that the larger grains are made out of smaller sub-grains. The average size of the holes is on the same order of magnitude as the 


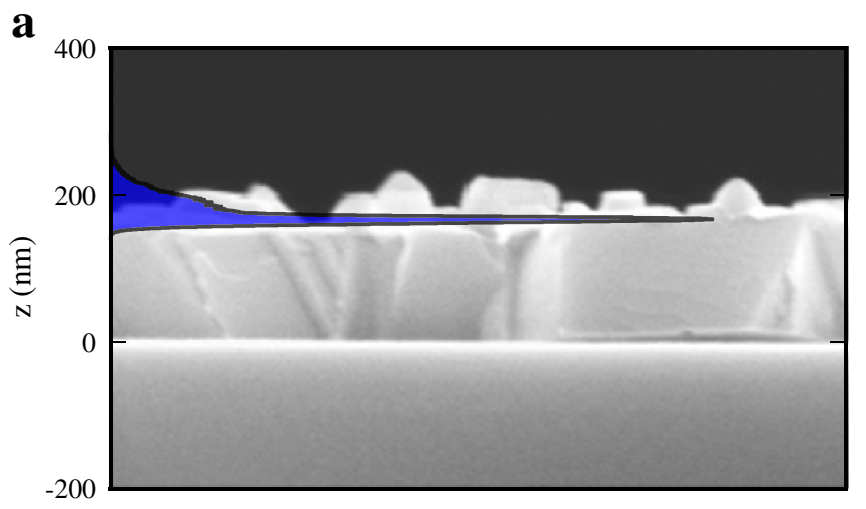

b



Fig. 6. SEM cross-section view of the deposited bismuth films. The histogram shows the distribution of $z$ values from the AFM measurement in Fig. 3b. The histogram is placed over the SEM image in such a way that the most frequently occurring value of $z$ is aligned with the apparent minimum film thickness in the SEM image.

grain-size observed on the top-side of the film and as the grain-size determined from the XRD rocking curve $(88 \mathrm{~nm})$.

The composite hexagonal shape of the holes that is clearly visible in Fig. 9c can be explained by three dimensional growth of nuclei [24]. The first bismuth atoms that arrive on the silicon surface combine into the smaller grains that we see in Fig. 9c. The grains fuse together to a maximum size that is determined by growth kinetics. Occasionally some atoms will bond not to the silicon surface, but to the sides of the islands of bismuth that are already present. In this way, new grains are formed on top of the bottom grains, preventing other atoms from arriving at the silicon surface. These places become the voids.

Surprisingly, in the $500 \mathrm{~nm}$ thick film, the size of the larger grains and the size of the smaller sub-grains are dissimilar to the size of the crystallites at the topside of the film. Observations made by Hattab et al. [15] on the structure of epitaxially grown films of bismuth provide a potential explanation. They observed that the first $6 \mathrm{~nm}$ of the bismuth film tends to match the silicon lattice. The lattice misfit is accommodated by 'an array of interfacial misfit dislocations'. After they deposited another layer of $19 \mathrm{~nm}$ on top of the existing film, they

\section{Table 2}

Thickness of the deposited bismuth films. The effective thickness is defined as the thickness the film would have had if it was perfectly flat, while containing the same amount of material.

\begin{tabular}{lll}
\hline & $\mathrm{t}_{\mathrm{d}}=200 \mathrm{~nm}$ & $\mathrm{t}_{\mathrm{d}}=500 \mathrm{~nm}$ \\
\hline thickness as measured in-situ & $208.6 \mathrm{~nm}$ & $507.8 \mathrm{~nm}$ \\
Apparent minimum thickness (SEM) & $(166 \pm 13) \mathrm{nm}$ & $(508 \pm 14) \mathrm{nm}$ \\
Effective thickness & $(176 \pm 13) \mathrm{nm}$ & $(526 \pm 14) \mathrm{nm}$ \\
\hline
\end{tabular}
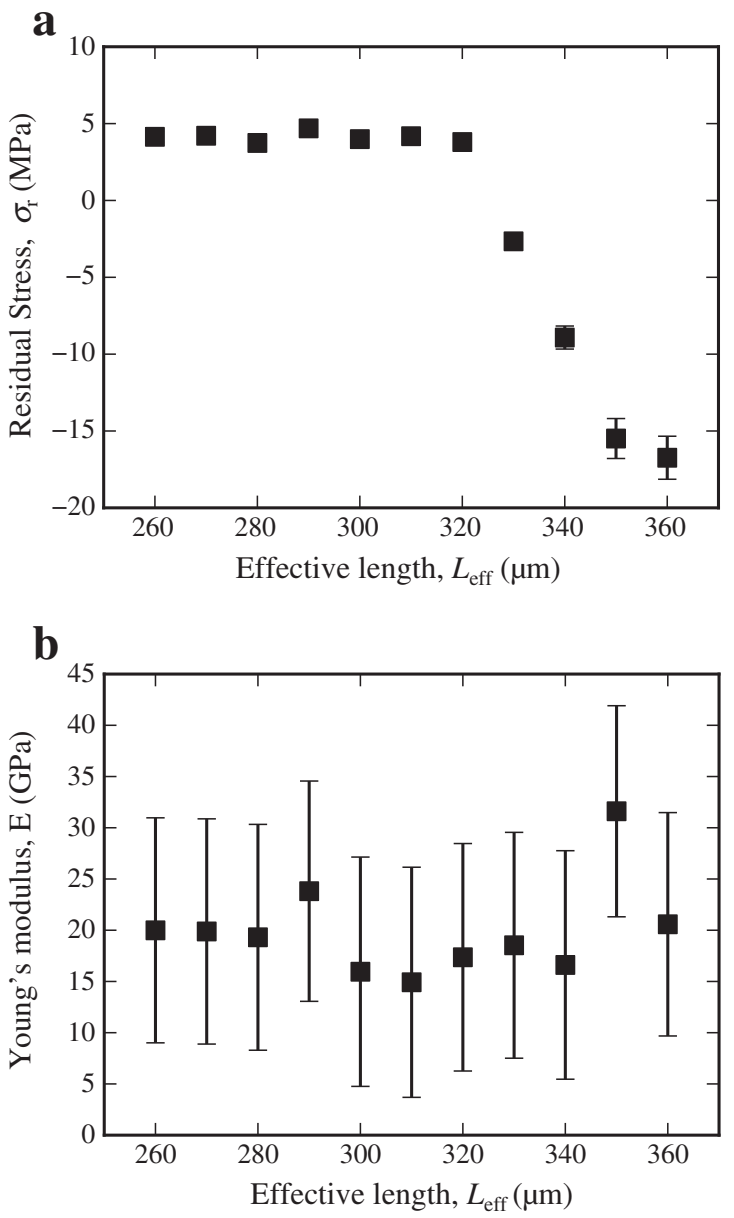

Fig. 7. The Young's modulus $E$ and residual stress $\sigma_{\mathrm{r}}$ of the $200 \mathrm{~nm}$ bismuth film against the effective cantilever length $L_{\text {eff. }}$
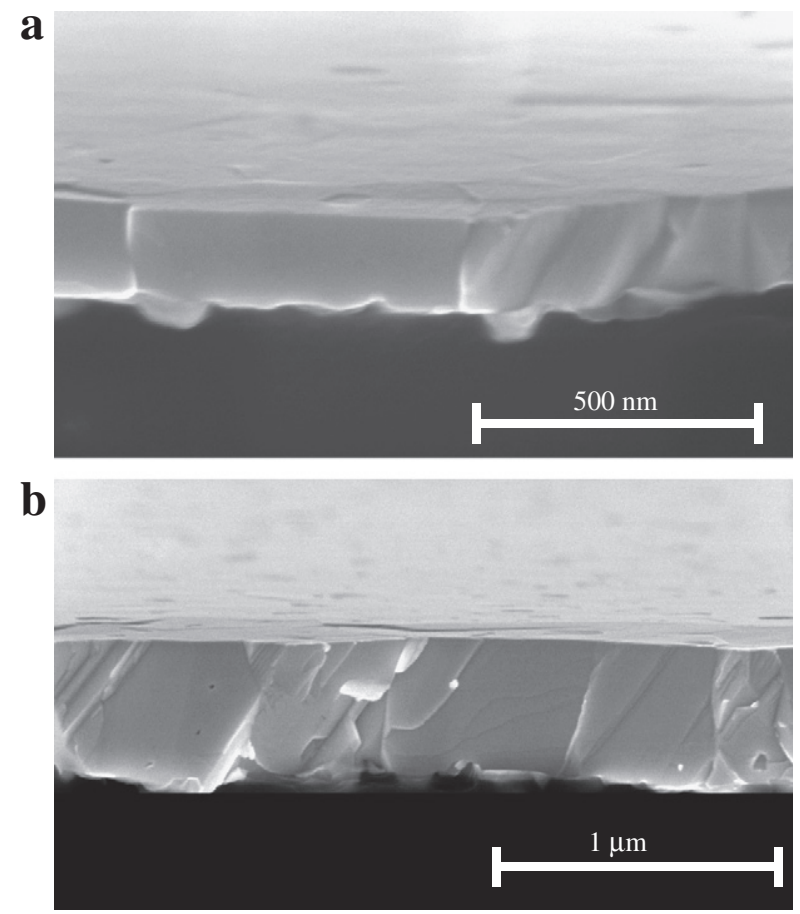

Fig. 8. Scanning electron micrographs of the film cross-sections after the film backsides have been revealed by template stripping. 

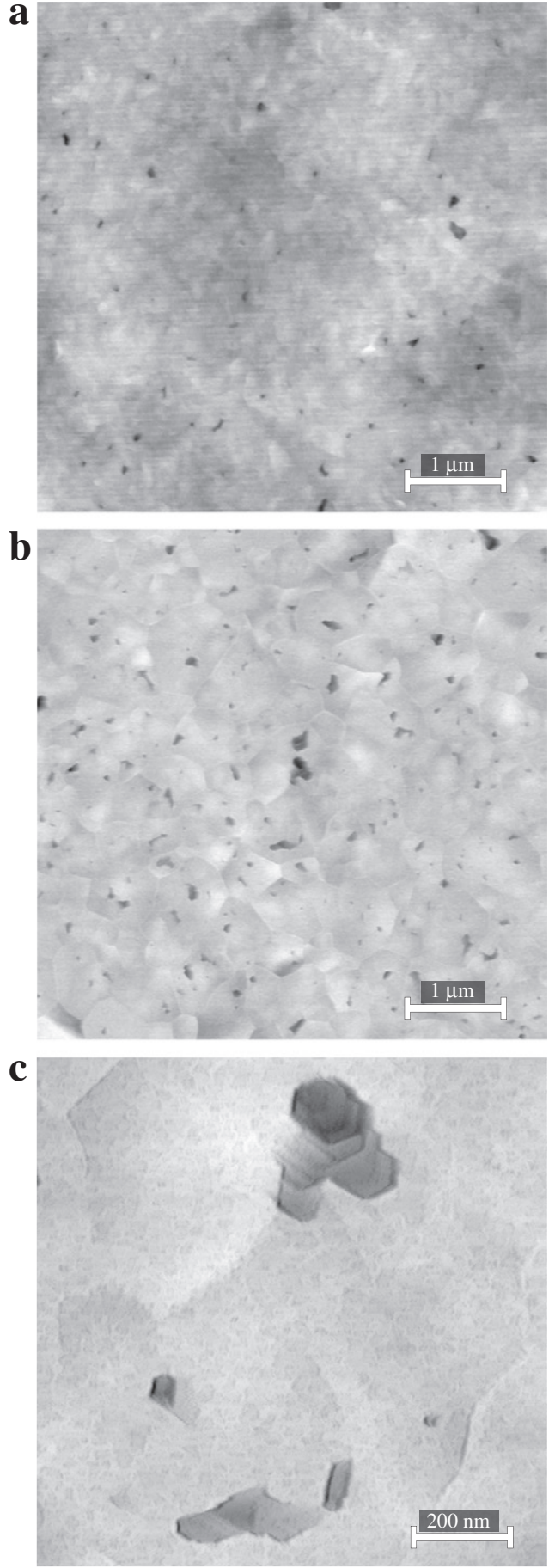

Fig. 9. AFM measurement of the smooth backsides of the thick films of bismuth.

observed that the film is relaxed to the bulk lattice constant. This means that the sub-grains combine into much larger grains higher up in the film. The stress between these larger grains is relaxed after stripping it

Table 3

Roughness parameters of the film backsides exposed by template stripping. The values are calculated from the $5 \mu \mathrm{m} \times 5 \mu \mathrm{m}$ AFM measurements shown in Fig. 9a and b.

\begin{tabular}{llc}
\hline & $\mathrm{t}_{\mathrm{d}}=200 \mathrm{~nm}$ & $\mathrm{t}_{\mathrm{d}}=500 \mathrm{~nm}$ \\
\hline $\mathrm{R}_{\mathrm{a}}$ & $0.58 \mathrm{~nm}$ & $0.54 \mathrm{~nm}$ \\
$\mathrm{R}_{\mathrm{pp}}$ & $9.27 \mathrm{~nm}$ & $11.94 \mathrm{~nm}$ \\
$\mathrm{R}_{\text {RMS }}$ & $0.74 \mathrm{~nm}$ & $0.79 \mathrm{~nm}$ \\
Median & $6.02 \mathrm{~nm}$ & $9.01 \mathrm{~nm}$ \\
Average & $6.00 \mathrm{~nm}$ & $8.96 \mathrm{~nm}$ \\
\hline
\end{tabular}

from the substrate. This relaxation results in the large grain structure that is visible in Fig. 9b.

\section{Conclusion}

Continuous films of thermally evaporated bismuth on silicon have a large surface roughness in comparison to their film thickness. SEM and AFM inspections of the film surface show a polycrystalline structure with an RMS roughness of $17.4 \mathrm{~nm}$ for a $200 \mathrm{~nm}$ thick film. The roughness increases to $25.5 \mathrm{~nm}$ for a $500 \mathrm{~nm}$ film. XRD measurements confirm that the films are polycrystalline, but highly textured with a preferential c-axis orientation.

By applying a template stripping method, the 'backside' of the film (the interface between the bismuth and the silicon) was exposed. The backside is smoother than the top by at least a factor 23. We obtained large areas up to several square centimeters of bismuth with an RMS surface roughness of $0.74 \mathrm{~nm}$ for the $200 \mathrm{~nm}$ thick film, and $0.79 \mathrm{~nm}$ for the $500 \mathrm{~nm}$ film.

AFM measurements of the backsides of the films show a fine grain structure of comparable dimensions in both films, but the grains of the $500 \mathrm{~nm}$ thick film are grouped into larger grains. We propose that this phenomenon is caused by stress relaxation of larger grains that exist higher-up in the film.

The Young's modulus of the $200 \mathrm{~nm}$ thick film was calculated to be $(20 \pm 11) \mathrm{GPa}$, which is on the order of the values reported for the different directions in bulk bismuth.

The template stripping method presented here solves the excessive surface roughness issue of thin bismuth films, and opens up the way towards diamagnetic levitation on the microscale.

\section{Acknowledgments}

We thank the MESA + cleanroom personnel, in particular Hans Mertens, Johnny Sanderink, Martin Siekman and Henk van Wolferen for their assistance and advice on thermal evaporation, and SEM and AFM imaging. We also would like to thank our colleagues at the Complex Photonic Systems (COPS) group for their advice and for letting us use their wafer bonding equipment. Last but not least, we would like to thank Ruud Hendrikx of the XRD Facilities Group at the TU Delft for his kind assistance on interpreting our XRD patterns.

\section{References}

[1] W. Braunbek, Z. Phys. 112 (1939) 764, http://dx.doi.org/10.1007/BF01339980.

[2] L. Schultz, O. De Haas, P. Verges, C. Beyer, S. Rölig, H. Olsen, L. Kühn, D. Berger, U. Noteboom, U. Funk, IEEE Trans. Appl. Supercond. 15 (2005) 2301, http://dx.doi.org/10.1109/TASC.2005.849636.

[3] W.M. Haynes (Ed.), CRC Handbook of Chemistry and Physics, Internet Version, 92nd ed., CRC Press/Taylor and Francis, Boca Raton, FL, USA, 2012.

[4] H.B. Profijt, C. Pigot, G. Reyne, R.M. Grechishkin, O. Cugat, J. Magn. Magn. Mater. 321 (2009) 259, http://dx.doi.org/10.1016/j.jmmm.2008.08.115.

[5] M.D. Simon, A.K. Geim, J. Appl. Phys. 87 (2000) 6200, http://dx.doi.org/10.1063/ 1.372654 .

[6] D. Garmire, H. Choo, R. Kant, S. Govindjee, C.H. Séquin, R.S. Muller, J. Demmel, in: Int. Solid-State Sensors, Actuators and Microsystems Conf., 2007, p. 1203, http://dx.doi.org/10.1109/SENSOR.2007.4300352.

[7] Ch. Pigot, G. Poulin, G. Reyne, Sens. Lett. 7 (2009) 329, http://dx.doi.org/10.1166/ sl.2009.1086.

[8] N.M. Dempsey, A. Walther, F. May, D. Givord, K. Khlopkov, O. Gutfleisch, Appl. Phys. Lett. 90 (2007) 092509, http://dx.doi.org/10.1063/1.2710771.

[9] A.W. Moore, in: K.H.J. Buschow, R.W. Cahn, M.C. Flemings, B.I. (print), E.J. Kramer, S. Mahajan, P.V. (updates) (Eds.), Encyclopedia of Materials: Science and Technology, Elsevier, Oxford, 2001, pp. 7933. http://dx.doi.org/10.1016/ B0-08-043152-6/01428-5.

[10] J.C.G. de Sande, T. Missana, C.N. Afonso, J. Appl. Phys. 80 (1996) 7023, http://dx.doi.org/10.1063/1.363775.

[11] D.-H. Kim, S.-H. Lee, J.-K. Kim, G.-H. Lee, Appl. Surf. Sci. 252 (2006) 3525, http://dx.doi.org/10.1016/j.apsusc.2005.05.046

[12] T. Missana, C.N. Afonso, Appl. Phys. A Mater. Sci. Process. 62 (1996) 513, http://dx.doi.org/10.1007/s003390050333.

[13] L. Kumari, S.-J. Lin, J.-H. Lin, Y.-R. Ma, P.-C. Lee, Y. Liou, Appl. Surf. Sci. 253 (2007) 5931, http://dx.doi.org/10.1016/j.apsusc.2006.12.125 
[14] V.M. Grabov, E.V. Demidov, V.A. Komarov, Phys. Solid State 50 (2008) 1365, http://dx.doi.org/10.1134/S1063783408070287.

[15] H. Hattab, E. Zubkov, A. Bernhart, G. Jnawali, C. Bobisch, B. Krenzer, M. Acet, R. Möller, M. Horn-von Hoegen, Thin Solid Films 516 (2008) 8227, http://dx.doi.org/10.1016/ j.tsf.2008.02.038.

[16] M. Hegner, P. Wagner, G. Semenza, Surf. Sci. 291 (1993) 39, http://dx.doi.org/ 10.1016/0039-6028(93)91474-4.

[17] S. Jeon, T. Thundat, Appl. Phys. Lett. 85 (2004) 1083, http://dx.doi.org/10.1063/ 1.1781389.

[18] H. Nazeer, M.D. Nguyen, L.A. Woldering, L. Abelmann, G. Rijnders, M.C. Elwenspoek, J. Micromech. Microeng. 21 (2011) 074008, http://dx.doi.org/10.1088/0960-1317/ 21/7/074008.
[19] W.A. Brantley, J. Appl. Phys. 44 (1973) 534, http://dx.doi.org/10.1063/ 1.1661935.

[20] G. Stoney, Proc. R. Soc. Lond. A 82 (1909) 172, http://dx.doi.org/10.1098/ rspa.1909.0021.

[21] P. Vereecken, L. Sun, P. Searson, M. Tanase, D. Reich, C. Chien, J. Appl. Phys. 88 (2000) 6529, http://dx.doi.org/10.1063/1.1323537.

[22] D. Gunton, G. Saunders, J. Mater. Sci. 7 (1972) 1061, http://dx.doi.org/10.1007 BF00550070.

[23] A. De Bretteville, E. Cohen, A. Ballato, I. Greenberg, S. Epstein, Phys. Rev. 148 (1966) 575, http://dx.doi.org/10.1103/PhysRev.148.575.

[24] S. Nakahara, Thin Solid Films 45 (1977) 421, http://dx.doi.org/10.1016/ 0040-6090(77)90229-2. 